\author{
MITSUBISHI ELECTRIC RESEARCH LABORATORIES \\ http://www.merl.com
}

\title{
Wireless Power Transfer with Artificial Magnetic Conductors
}

Wu, J.; Wang, B.; Yerazunis, W.S.; Teo, K. H.

TR2013-018 May 2013

\begin{abstract}
In this paper, a wireless power transfer system (WPT) with magnetically coupled resonators is studied. The idea to use a reflector to enhance the coupling coefficient and the transfer efficiency is proposed and analyzed. Perfect magnetic conductor (PMC) was used in numerical calculations in order to study the performance of a reflector. In the WPT system we studied, efficiency is increased from $48 \%$ without reflector to $72 \%$ with PMC reflector. With recently developed metamaterial technologies, artificial PMCs can be designed and fabricated for such reflectors.
\end{abstract}

IEEE Wireless Power Transfer Conference (WPTC)

This work may not be copied or reproduced in whole or in part for any commercial purpose. Permission to copy in whole or in part without payment of fee is granted for nonprofit educational and research purposes provided that all such whole or partial copies include the following: a notice that such copying is by permission of Mitsubishi Electric Research Laboratories, Inc.; an acknowledgment of the authors and individual contributions to the work; and all applicable portions of the copyright notice. Copying, reproduction, or republishing for any other purpose shall require a license with payment of fee to Mitsubishi Electric Research Laboratories, Inc. All rights reserved.

Copyright (C) Mitsubishi Electric Research Laboratories, Inc., 2013

201 Broadway, Cambridge, Massachusetts 02139 



\title{
Wireless Power Transfer with Artificial Magnetic Conductors
}

\author{
Jing Wu, Bingnan Wang, William S. Yerazunis, Koon Hoo Teo \\ Mitsubishi Electric Research Laboratories, 201 Broadway, Cambridge, MA 02139, USA \\ Email: bwang@merl.com
}

\begin{abstract}
In this paper, a wireless power transfer system (WPT) with magnetically coupled resonators is studied. The idea to use a reflector to enhance the coupling coefficient and the transfer efficiency is proposed and analyzed. Perfect magnetic conductor (PMC) was used in numerical calculations in order to study the performance of a reflector. In the WPT system we studied, efficiency is increased from $48 \%$ without reflector to $72 \%$ with PMC reflector. With recently developed metamaterial technologies, artificial PMCs can be designed and fabricated for such reflectors.

Index Terms - Wireless power transfer (WPT); metamaterials; artificial perfect magnetic conductor (APMC).
\end{abstract}

\section{Introduction}

In recent years, considerable effort has been put into the research of wireless power transfer technologies, specifically for cordless devices, such as low power consumer electronics and implanted medical devices, as well as high-power industrial and electric/hybrid vehicles. WPT was first pursued by Tesla over 100 years ago. However, safety, efficiency, and other issues have prevented WPT from deployment in real applications in daily life. Recent research efforts have been focused on technologies based on magnetic coupling [1]-[4], as they are less sensitive to surrounding objects and safer for humans, and more suitable for the increasing demands for wireless charging of electronic devices. With magnetic coupling or inductive coupling, very high efficiency between transmitting and receiving coils can be achieved at small distances of less than a few centimeters [6]. Recently, WPT over a distance up to 2 meters with moderate efficiency and power level has been achieved [1]. Furthermore, various approaches have been proposed to enhance the transfer efficiency of the WPT system. It has been demonstrated that a metamaterial slab, when placed between the source and sink coil can enhance the evanescent field distribution, and therefore increase the transfer efficiency [7]-[11]. However, in order to achieve the optimal performance, the metamaterial slab is usually placed at the center of the space, which is not very convenient for some applications.

In this paper, the use of a reflector to enhance the coupling coefficient and the transfer efficiency is proposed and analyzed. In particular, the metamaterial known as perfect magnetic conductor (PMC) is studied and used as reflector in a WPT system. Numerical simulations are done to confirm that the efficiency of WPT is increased from $48 \%$ to $72 \%$ with PMC reflectors. Although PMCs do not exist in nature, they can be engineered with array of resonant structures using recently developed metamaterial technologies.

\section{Image Theory for Coil with Reflector}

In order to improve near-field magnetic field based WPT, we want to improve the magnetic field coupled from transmitting coil to receiving coil. We thus need something that can provide a form of confinement to the magnetic field, to prevent the magnetic field from the resonators from going in the opposite direction from the receiving coil, and enhance the magnetic field in the direction of the receiving coil. From image theory in electromagnetics, a PMC can achieve these goals.

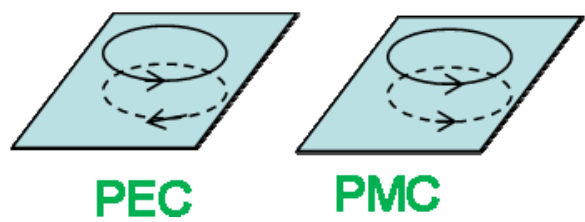

Fig. 1. A current carrying loop and image currents for (a) PEC plane, and (b) PMC plane.

The proposed idea was first investigated with a simple coil current source with radius $r$, located close to a reflector, as shown in Fig.1. According to the image theory, there is image current at the other side of the plane. For perfect electric conductor (PEC), the image current is reversed in direction from the originating

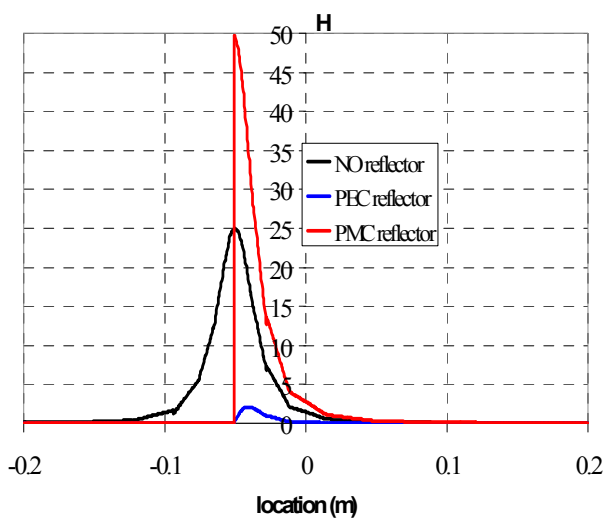

Fig. 2. Magnetic field distribution versus distance from the loop source, calculated with COMSOL. Note that for an ideal PMC, the magnetic field is zero on the far side of the coil. 
current flow; thus the image current generates an opposing magnetic field, as expected by Lenz's law. On the other hand, for PMC reflector, the image current has the same direction as the original current flow, and the resulting magnetic field is reinforced. These image currents can contribute to the enhancement of evanescent field distribution. In our tests, the loop current source was located at $\mathrm{z}=-0.05 \mathrm{~m}$, with radius $\mathrm{r}=0.02 \mathrm{~m}$. The reflector was located at $\mathrm{z}=-0.051 \mathrm{~m}$. Fig. 2 shows magnetic field versus distance from the loop source, where the magnitude of magnetic field was almost doubled within the distance of a few times of the loop radius, which may enhance the coupling if a receive coil is placed in this range.

It is important to realize that although the image current in a PMC is in the same direction as the current in the transmitting coil, the image current does not cause a magnetic field on the far side of the PMC from the loop source. Rather, the PMC in effect "reflects" the magnetic field, and in the ideal case, the magnetic field on the far side of the PMC is zero.

\section{WPT System with PMC Reflector}

To confirm these concepts, we investigated a wireless power transfer system based on magnetic resonant coupling. The system is based on coupled spiral resonant coils, as shown in Fig. 3. The parameters of the resonant
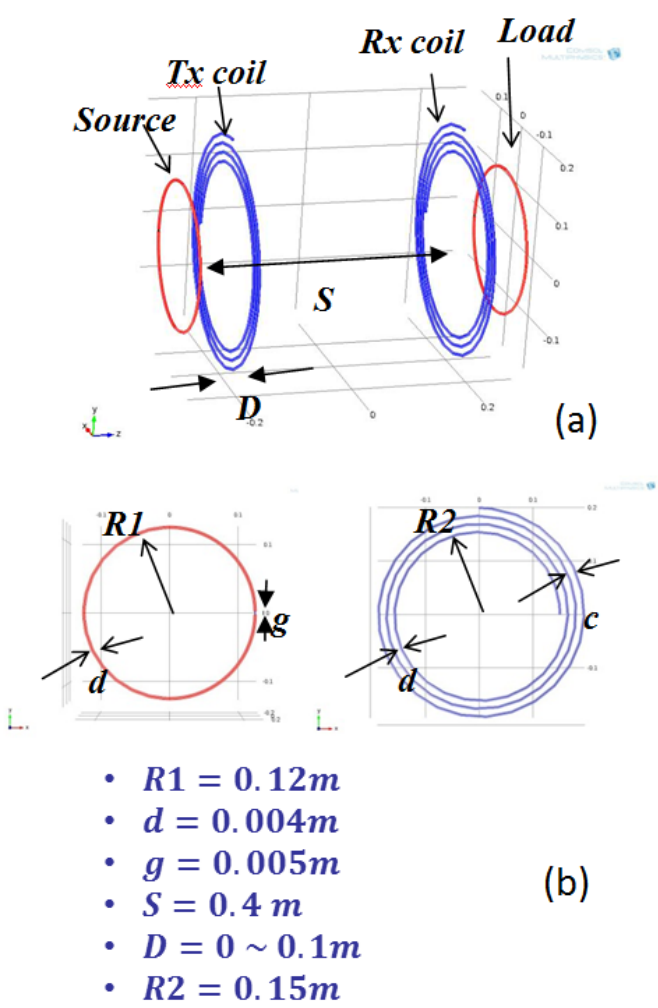

Fig. 3. (a) The resonant coupling based WPT system used in numerical studies; (b) Geometry of non-resonant loop and resonant coil in the WPT system, and corresponding parameters.

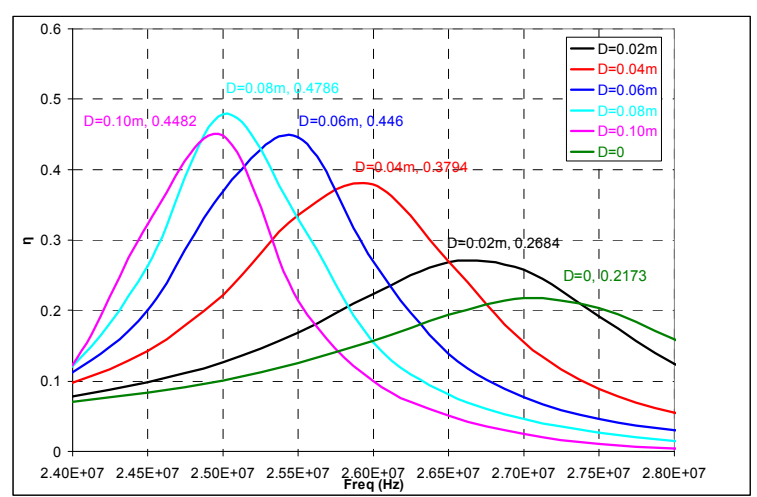

Fig. 4. Geometry of the advanced one coil to one coil WPT system with close PMC.

coils and the relative positions are also given in the figure. Tx and Rx spiral coils have a self-resonance at around 25 $\mathrm{MHz}$. The single turn power and load loops are nonresonant at that frequency range. The RF power is provided to the single turn power loop, which feeds the source coil resonator inductively. The source coil is coupled resonantly to a sink resonator and a load loop is used to extract power from the sink resonator inductively.

The system is modeled and calculated with COMSOL, which is a commercial simulation package based on finiteelement method. The efficiency is calculated as the ratio of the power extracted by the load loop divided by the power injected at the power loop. Since a major figure of merit of a wireless power system is the efficiency at a particular unobstructed gap, we tested with a constant $\mathrm{S}$ (unobstructed distance between two resonant coils) and varied the distance from power loop to Tx coil, and the distance from $\mathrm{Rx}$ coil to load loop. This allows an optimization of power transfer efficiency. In the studied system, D is varied from 0 to $10 \mathrm{~cm}$, and the efficiency as a function of frequency is calculated for each case, and plotted in Fig. 4. An optimal matching is achieved when D is $8 \mathrm{~cm}$. For a transfer distance $\mathrm{S}=0.4 \mathrm{~m}$, an optimal efficiency $47.86 \%$ is achieved at frequency $25 \mathrm{MHz}$.

The frequency response of the efficiency shown in Fig. 4 is typical for resonant coupling based WPT. At peak of the curve, resonant coupling between two resonant coils is achieved, and energy exchange rate is optimal [1]. No

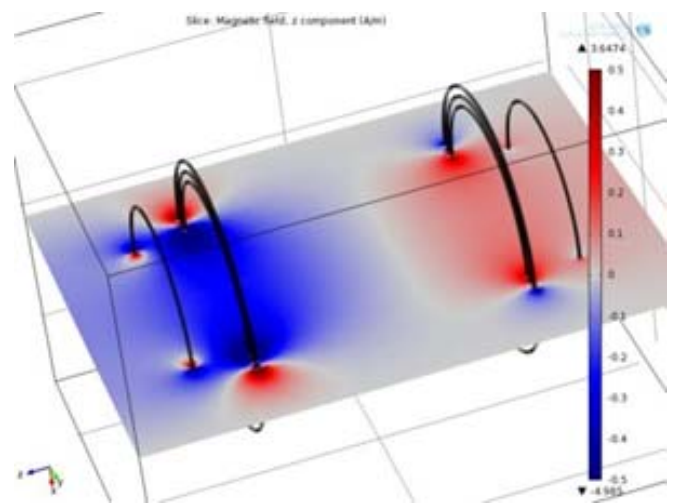

Fig. 5. Magnetic field distribution of the WPT system when $\mathrm{D}=8 \mathrm{~cm}$ and $\mathrm{f}=25 \mathrm{MHz}$. 


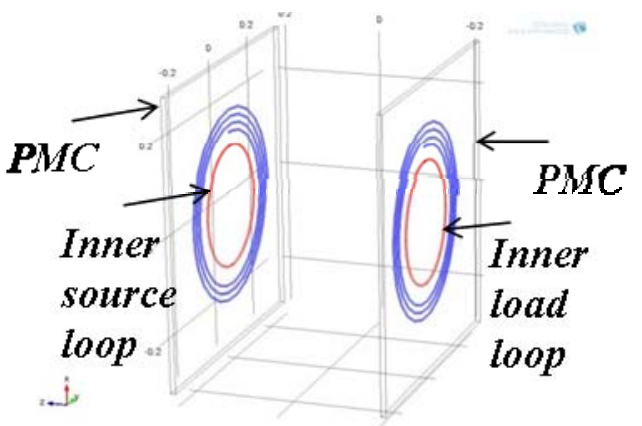

Fig. 6. Geometry of the WPT system with PMC reflectors on both sides

frequency splitting is observed in this configuration, indicating that the system is under coupled. A simulated field distribution at the optimal condition $(D=8 \mathrm{~cm}, f=25$ $\mathrm{MHz}$ ) shown in Fig. 5 indicates the resonance behavior where strong field is localized in both transmitting and receiving coils even though the field is very weak in between.

For real applications, we want the system as compact and thin as possible. The optimized case without PMC has a distance of $8 \mathrm{~cm}$ between power loop and resonant coil, which is rather thick for practical implementation. The ideal case is to have the power loop and resonant coil in the same plane so that they could be put together with a thin package. In this case, as shown in Fig. 4, when $D=0$ (loop and corresponding coil share the same plane), the system is not matched and efficiency is only $21.73 \%$.

In order to study the effect of PMC reflector and the ability of PMC to produce a very thin, easily inserted WPT system, we modified the WPT system configuration, placing the non-resonant loop inside corresponding coil so they share the same plane. A PMC plane is placed directly behind each coil and loop, with a spacing of $0.6 \mathrm{~cm}$, as shown in Fig. 6. This decreases the thickness of both the transmitting and receiving systems from $8 \mathrm{~cm}$ to $1 \mathrm{~cm}$ thick. The unobstructed distance between two resonant coils is still $\mathrm{S}=40 \mathrm{~cm}$.

Simulations were done for this configuration to calculate the power transfer efficiency. Fig.7 shows the

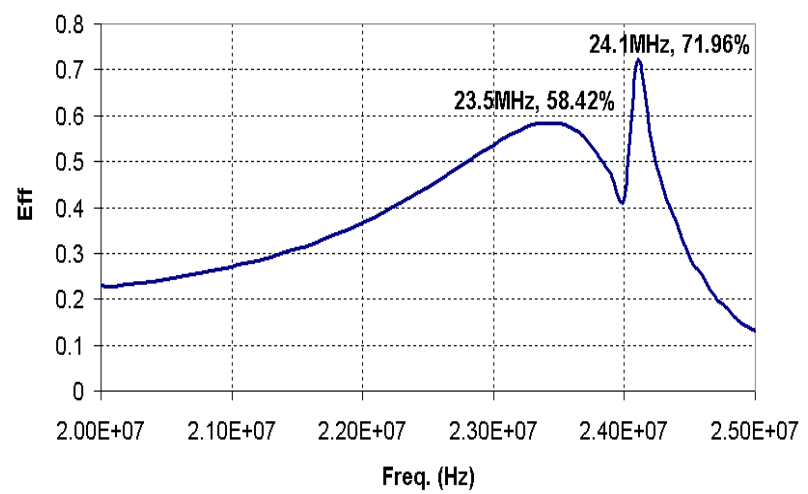

Fig. 7. Power transfer efficiency of the WPT system with $\mathrm{PMC}$ as reflectors.
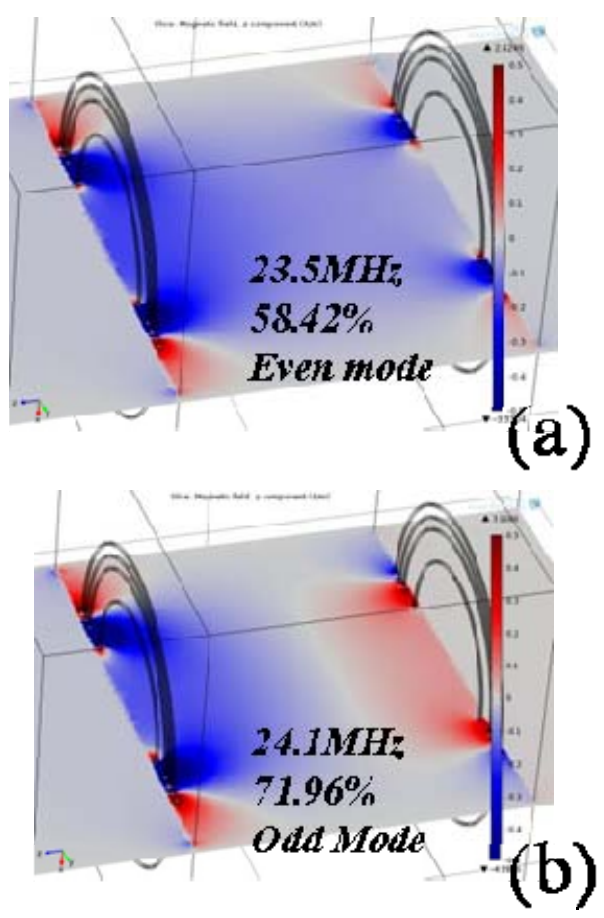

Fig. 8. Magnetic field distribution of the WPT system with PMC as reflectors for (a) even mode and (b) odd mode. Frequency and corresponding power transfer efficiency are given in insets.

transfer efficiency of this system as a function of frequency. The efficiency increases to $71.96 \%$ from $47.86 \%$ at $24.1 \mathrm{MHz}$, which is a very promising enhancement. Also, we can see frequency splitting happens around $24 \mathrm{MHz}$, which is an indication of stronger coupling between resonant coils. In other words, the coupling coefficient of the WPT system has been enhanced by the PMC reflectors. As expected, the reflectors modify the self inductance and capacitance of the resonant coils and thus change their resonant frequency.

Fig. 8 shows the simulated magnetic field distribution for even and odd modes, respectively. We can see that the fields are reflected by the PMC planes, and more field is coupled to the receiving side.

Since perfect magnetic conductors do not exist in nature, they must be artificially created. With recently developed metamaterial and other related technologies, there are ways to build such magnetic conductors. For example, a band-limited artificial PMC can be created by placing periodic elements such as square conductive patches with central conducting vias through an insulating substrate connecting to a conducting backplane (sometimes called the "mushroom array" configuration). These periodic arrays can be modified by adding spirals, inductors, etc. to modify the frequency response to the frequency band desired. Artificial PMCs can be designed and fabricated depending on the frequency and size requirements and fit into the WPT system. 
Other than the much improved power transfer performance with PMC reflector, another obvious advantage of this configuration is that the transmitting device and receiving device can be made very compact and thin; and there is no need to place any special material into the gap between the resonators, which increases the practical applications of this method. The PMC reflectors serve as electromagnetic field shields for the system, confining the strong magnetic field of the WPT system, which helps to improve the RF safety of the WPT system, which is especially desirable for high power applications. This makes the system more practical to implement for various applications.

We should temper the quality of these results by noting that this improvement is with an ideal PMC. A real PMC implemented with metamaterial techniques will at least have resistive losses in the conductive elements, as well as a nonzero leakage of magnetic field through the material. Fortunately, the experience in previous references $[7,8$, $10,11]$ indicates that these losses in artificially engineered materials can be managed to be small compared to the efficiency gains.

\section{Conclusions}

In conclusion, we studied a wireless power transfer system based on resonant coupling and investigated the idea of using special reflectors to enhance the coupling and the power transfer efficiency. In particular, we studied the case of perfect magnetic conductor material used as reflectors and their impact on WPT. We showed with numerical simulations that the WPT efficiency can be increased from $48 \%$ without reflector to $72 \%$ with PMC reflectors, at a distance between coils of $40 \mathrm{~cm}$, at the same time decreasing the thickness of each endpoint from $8 \mathrm{~cm}$ to $1 \mathrm{~cm}$. With the advantages of compact size, high efficiency, and lost-field shielding, the system with PMC reflectors is very promising for many applications.

\section{References}

[1] A. Kurs, A. Karalis, R. Moffatt, J. D. Joannopoulos, P. Fisher and M. Soljiacic, "Wireless power transfer via strongly coupled magnetic resonances," Science, 317, 83 (2007)

[2] Z. N. Low, R. A. Chinga, R. Tseng and J. Lin, "Design and Test of a High-Power High-Efficiency Loosely Coupled Planar Wireless Power Transfer System," IEEE Transactions on Industrial Electronics, 56, 1801 (2009)

[3] B. L. Cannon, J. F. Hoburg, D. D. Stancil and S. C. Goldstein, "Magnetic Resonant Coupling As a Potential Means for Wireless Power Transfer to Multiple Small Receivers," IEEE TRANSACTIONS ON POWER ELECTRONICS, 24, 1819, (2009)

[4] G. A. J. Elliott, S. Raabe, G. A. Covic, and J. T. Boys, "Multiphase Pickups for Large Lateral Tolerance Contactless Power-Transfer Systems," IEEE TRANSACTIONS ON INDUSTRIAL ELECTRONICS, 57,1590 (2010)

[5] N. Shimokura, N. Kaya, M. Shinohara, and H. Matsumoto, "point-topoint microwave power transmission experiment" Electrical Engineering in Japan 120 (1), 33-39 (1997)

[6] J. C. Schuder, H. E. Stephenson and J. F. Townsend, "Highlevel electromagnetic energy transfer through a closed chest wall," Inst. Radio Engrs. Int Conv. Record, 9, 119 (1961)

[7] B. Wang, T. Nishino, K. H. Teo, "Wireless Power Transmission Efficiency Enhancement with Metamaterials", IEEE International Conference on Wireless Informatio Technology and Systmes, August 2010

[8] B. Wang, K. H. Teo, T. Nishino, W. Yerazunis, J. Barnwell, and J. Zhang, "Wireless Power Transfer with Metamaterials," in Proceedings of European Conference on Antennas and Propagation (EuCAP 2011) (April 11-15 2011, Rome, Italy)

[9] Y. Urzhumov and D. R. Smith, "Metamaterial-enhanced coupling between magnetic dipoles for efficient wireless power transfer," Phys. Rev. B 83, 205114 (2011)

[10] B. Wang, K. H. Teo, T. Nishino, W. Yerazunis, J. Barnwell and J. Zhang, "Experiments on wireless power transfer with metamaterials," Appl. Phys. Lett. 98, 254101 (2011)

[11] B. Wang, W. Yerazunis, and K. H. Teo, "Wireless power transfer: metamaterials and array of coupled resonators," accepted to the Proceedings of the IEEE 